Article

\title{
Facile Synthesis of Functionalized Spiropyrrolizidine Oxindoles via a Three-Component Tandem Cycloaddition Reaction
}

\author{
Yong-Mei Xie ${ }^{1}$, Yu-Qin Yao ${ }^{1}$, Hong-Bao Sun ${ }^{1}$, Ting-Ting Yan ${ }^{1}$, Jie Liu ${ }^{1, *}$ and \\ Tai-Ran Kang ${ }^{2, *}$
}

1 State Key Laboratory of Biotherapy, West China Hospital, West China Medical School, Sichuan University, Chengdu, 610041, China; E-Mails: xieym@scu.edu.cn (Y.M.X.); yuqin_yao@163.com (Y.Q.Y.); 773770248@qq.com (H.B.S.); 121968698@qq.com (T.T.Y.)

2 Chemical Synthesis and Pollution Control Key Laboratory of Sichuan Province, College of Chemistry and Chemical Engineering, China West Normal University, Nanchong, 637002, China

* Authors to whom correspondence should be addressed; E-Mails: liujie2011@scu.edu.cn (J.L.); kangtairan@cwnu.edu.cn (T.R.K.); Tel.: +86-28-85503817; Fax: +86-28-85503817.

Received: 26 August 2011; in revised form: 30 September 2011/ Accepted: 3 October $2011 /$

Published: 19 October 2011

\begin{abstract}
An efficient synthesis of functionalized spiropyrrolizidine oxindoles via a threecomponent tandem cycloaddition has been achieved. This strategy can provide direct and rapid access to spiropyrrolizidine oxindoles in high yields (up to 99\%) with excellent diastereoselectivities (up to 99:1 dr). The features of this procedure are the following: mild reaction conditions, high yields, high diastereoselectivities, one-pot procedure and operational simplicity.
\end{abstract}

Keywords: spiropyrrolizidine oxindoles; cycloaddition; isatin

\section{Introduction}

Spirocyclic oxindoles are valuable synthetic intermediates and constitute the core units of many pharmacological agents and alkaloids [1-3]. These compounds have attracted much attention from synthetic chemists due to their diverse biological activities including antimycobacterial [4-9], antitumor [10-14], antimicrobial [15], antibacterial [16,17], antifungal [18,19], antiviral [20,21], and local anesthetic [22] properties. Hence, a number of synthetic routes have been developed for the preparation of these structural frameworks [23-33]. 1,3-Dipolar cycloaddition provides an efficient 
approach for the synthesis of five-membered heterocycles [34,35] and spiro-heterocycles, such as poly functionalized pyrrolidines [36-39], pyrazolidines and pyrrolizines [40,41], which widely occur in natural products and biologically active compounds. Although there are reports of synthesis of these substituted heterocycles, the development of synthetically important functionalized new spiroheterocycles is still a challenge and has become a much attempted research endeavor.

Spiropyrrolizidine oxindoles are important synthetic targets and several reports of such syntheses exist $[42,43]$. To the best of our knowledge, however, there are no reports concerning the synthesis of spiropyrrolizidine oxindoles 4 containing two ester groups or two amide groups, which could possess some interesting biological activities. Herein, we report a three-component tandem cycloaddition reaction between substituted isatins, L-proline and maleates (maleamide) that produces such structures.

\section{Results and Discussion}

From the mechanistic perspective, the azomethine ylides, a class of powerful reagents, have emerged in a number of 1,3-dipolar cycloaddition reactions. In combination with the experiences in previous work, we envisaged that an azomethine ylide could be generated in situ from isatin (1a) and L-proline (2), and then trapped with dimethyl maleate (3a) acting as dipolarophile to afford spiropyrrolizidine oxindole 4a. Hence, the 1,3-dipolar cycloaddition reaction would be facilitated (Scheme 1).

Scheme 1. Possible reaction mechanism for the synthesis of spiropyrrolidine oxindole.

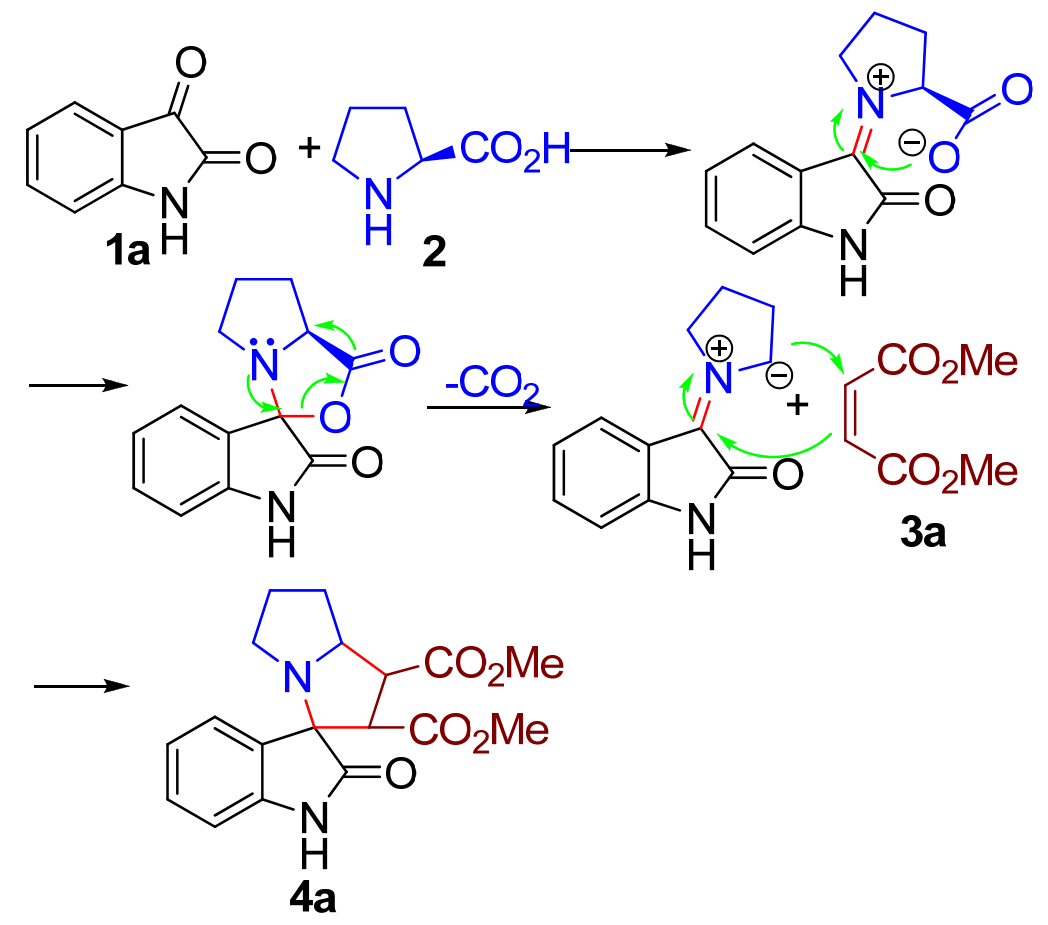

In light of the above considerations, the reaction in methanol at $60{ }^{\circ} \mathrm{C}$ of dimethyl maleate with azomethine ylide (generated in situ by decarboxylative condensation of isatin and L-proline) was examined. After $3 \mathrm{~h}$, the expected adduct $4 \mathrm{a}$ was obtained in $87 \%$ yield (Table 1, entry 1). We were pleased to see that our reaction afforded the adduct $4 \mathbf{a}$ with excellent diastereoselectivity (99:1, determined by $\left.{ }^{1} \mathrm{H}-\mathrm{NMR}\right)$. The structure of $\mathbf{4 a}$ was further confirmed by a single crystal X-ray 
crystallographic study (Figure 1) [14]. The ORTEP diagram of 4a shows that: (i) the pair of linked pyrrole rings of pyrrolizidine nucleus adopts an envelope-like conformation, (ii) H-3, H-4 and H-5 are all cis and (iii) the two carbonyls linked to C-2 and C-3 of 4a have a trans stereochemical relationship. This can be explained by the fact that the corresponding endo transition state (A) would require less free energy of activation than the exo transition state (B) leading to $\mathbf{4} \mathbf{a}^{\prime}$ as the latter would result in electrostatic repulsion between the cis carbonyls increasing the free energy of activation (Scheme 2). Therefore, the relative configuration of $\mathbf{4 a}$ was assigned as shown in Table 1.

Table 1. Optimization of reaction conditions ${ }^{\text {a }}$.

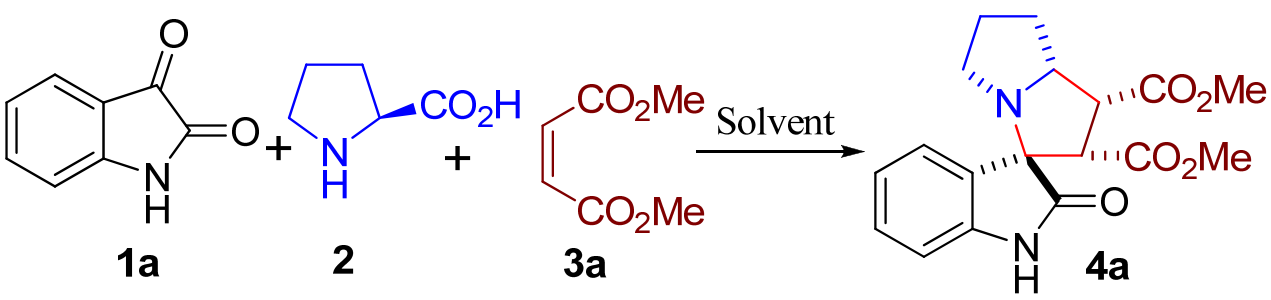

\begin{tabular}{llcc}
\hline Entry & Solvent & Temp $/{ }^{\circ} \mathbf{C}$ & Yield $/ \mathbf{\%}^{\mathbf{b}}$ \\
\hline 1 & methanol & 60 & 87 \\
2 & ethanol & 60 & 63 \\
3 & isopropanol & 60 & 71 \\
4 & acetonitrile & 60 & 82 \\
5 & chloroform & 60 & 84 \\
6 & tetrahydrofuran & 60 & 94 \\
7 & 1,4-dioxane & 60 & 99 \\
8 & 1,4-dioxane & 25 & 37 \\
$9^{\text {c }}$ & 1,4-dioxane & reflux & 94 \\
\hline
\end{tabular}

${ }^{a}$ Unless indicated otherwise, the reaction was carried out in $0.2 \mathrm{mmol}$ scale in solvent $(1 \mathrm{~mL})$ at $60{ }^{\circ} \mathrm{C}$ for $3 \mathrm{~h}$, and the ratio of $\mathbf{1 a / 2} / \mathbf{3 a}$ is $1: 1: 1$. $^{\mathrm{b}}$ Isolated yield based on isatin. ${ }^{\mathrm{c}} 45 \mathrm{~min}$.

Figure 1. X-ray structure of racemic compound $4 \mathbf{a}$.

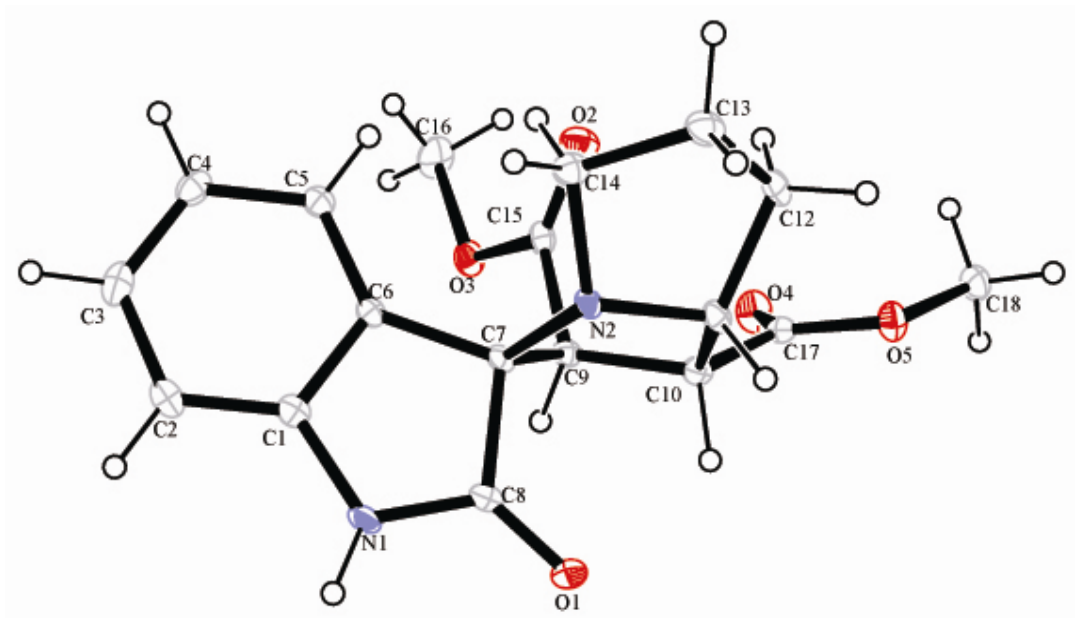


Scheme 2. Stereochemistry of cycloadducts differing in their relative configuration.

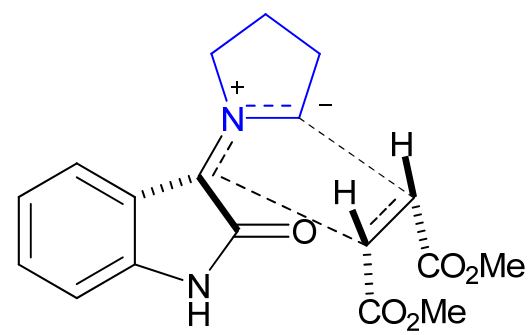

Endo-TS A

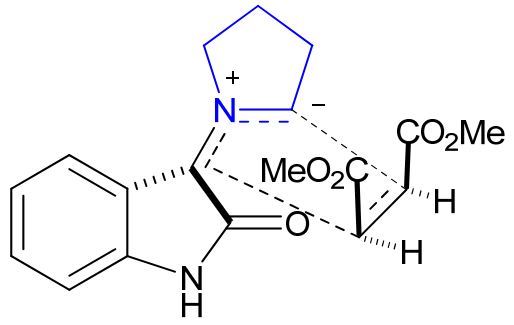

Exo-TS B

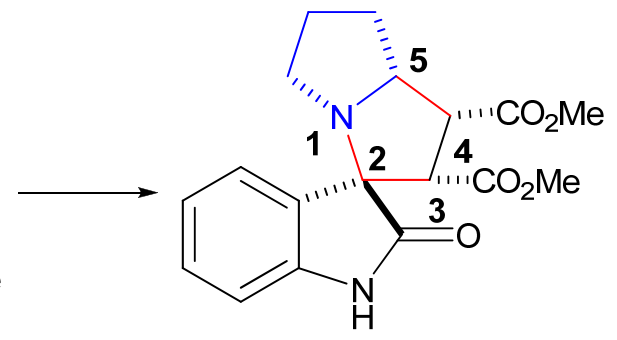

endo racemic 4a (major)

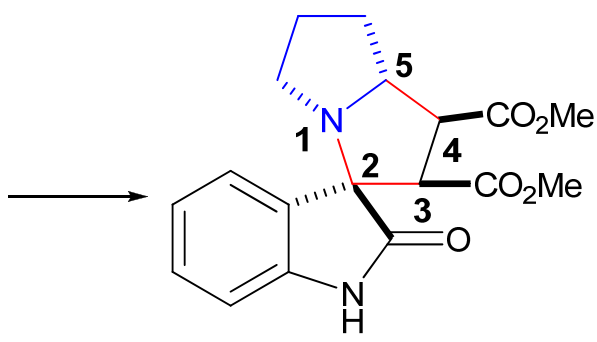

exo racemic 4a' (minor)

To improve the yield, efforts were made to optimize other reaction parameters including solvents and reaction temperatures. Thus, the reaction was studied in different solvents that included ethanol, isopropanol, acetonitrile, chloroform, tetrahydrofuran and 1,4-dioxane (Table 1, entries 2-7). To our satisfaction, the reaction in 1,4-dioxane led to the desired product in almost quantitative yield (99\%) and maintained stereoselectivity (Table 1, entry 7), while ethanol as solvent gave the product in only $63 \%$ yield (Table 1, entry 2). In general, reactions carried out in aprotic solvents were better yielding than those in protic solvents. Temperature influenced the rate of the reaction. Elevating the reaction temperature resulted in a high reactivity and the reaction time was shortened to $45 \mathrm{~min}$ (Table 1, entry 9). Based on the consideration of reaction time and yield, the optimized conditions were those shown in Table 1 , entry 7.

To show the general nature of the reaction, isatin bearing different substituents and L-proline were reacted with maleates (maleamide) under optimized conditions. Various functional groups appeared to be well tolerated and gave the corresponding spiropyrrolizidine oxindoles in moderate to good yields (51-99\%) with excellent diastereoselectivities (up to 99:1). The results are summarized in Table 2. For dimethyl maleate, the results showed that the reaction took place with excellent diastereo-selectivities of up to 99:1, regardless of the electronic and steric nature of the substituted isatins. However, the yields of the reaction were affected by the substitutent group on the isatins (Table 2, entries 1-6). 5-Bromoisatin resulted in low to 71\% yield, with an extention of the reaction time to $8 \mathrm{~h}$ (Table 2, entry 3). The oxindole core may also be modified. Thus, the N-protecting group may be changed as well. Incorporating different protecting groups on the N1 of oxindole had little effects on reactivity and diastereoselectivity (Table 2, entries 7-8). For the diethyl maleate, a similar phenomenon was observed. Substituents on the isatins influenced the diastereoselectivities only slightly, but affected the yields to a greater extent (Table 2, entries 9-16). Generally, isatins with electron-withdrawing groups gave lower yields than those with electron-donating groups. However, when we further expanded the substrate scope to maleamide (Table 2, entries 17-18), the corresponding products were obtained in 
moderate yields (51-64\%). The stereochemistry of the other products was assigned by analogy to the relative configuration of $\mathbf{4 a}$.

Table 2. Synthesis of racemic spiropyrrolizidine oxindoles ${ }^{\mathrm{a}}$.

\begin{tabular}{|c|c|c|c|c|c|c|}
\hline Entry & 1 & 3 & 4 & Time / h & Yield / \% ${ }^{b}$ & $d r^{c}$ \\
\hline 1 & $\mathrm{R}_{1}=\mathrm{R}_{2}=\mathrm{R}_{3}=\mathrm{H}$ & $\mathrm{R}_{4}=\mathrm{Me}$ & $4 \mathbf{a}$ & 3 & 99 & $99 / 1$ \\
\hline 2 & $\mathrm{R}_{1}=\mathrm{R}_{2}=\mathrm{H}, \mathrm{R}_{3}=\mathrm{CH}_{3}$ & $\mathrm{R}_{4}=\mathrm{Me}$ & $4 \mathbf{b}$ & 2 & 95 & $97 / 3$ \\
\hline 3 & $\mathrm{R}_{1}=\mathrm{R}_{2}=\mathrm{H}, \mathrm{R}_{3}=\mathrm{Br}$ & $\mathrm{R}_{4}=\mathrm{Me}$ & $4 c$ & 8 & 71 & $95 / 5$ \\
\hline 4 & $\mathrm{R}_{1}=\mathrm{H}, \mathrm{R}_{2}=\mathrm{R}_{3}=\mathrm{F}$ & $\mathrm{R}_{4}=\mathrm{Me}$ & $\mathbf{4 d}$ & 3 & 90 & $99 / 1$ \\
\hline 5 & $\mathrm{R}_{1}=\mathrm{H}, \mathrm{R}_{2}=\mathrm{R}_{3}=\mathrm{Cl}$ & $\mathrm{R}_{4}=\mathrm{Me}$ & $4 e$ & 3 & 89 & $99 / 1$ \\
\hline 6 & $\mathrm{R}_{1}=\mathrm{R}_{2}=\mathrm{H}, \mathrm{R}_{3}=\mathrm{COOH}$ & $\mathrm{R}_{4}=\mathrm{Me}$ & $\mathbf{4 f}$ & 3 & 95 & $99 / 1$ \\
\hline 7 & $\mathrm{R}_{1}=\mathrm{Et}, \mathrm{R}_{2}=\mathrm{R}_{3}=\mathrm{H}$ & $\mathrm{R}_{4}=\mathrm{Me}$ & $4 g$ & 3 & 88 & $99 / 1$ \\
\hline 8 & $\mathrm{R}_{1}=$ benzyl, $\mathrm{R}_{2}=\mathrm{R}_{3}=\mathrm{H}$ & $\mathrm{R}_{4}=\mathrm{Me}$ & $4 h$ & 2 & 93 & $99 / 1$ \\
\hline $9^{c}$ & $\mathrm{R}_{1}=\mathrm{R}_{2}=\mathrm{R}_{3}=\mathrm{H}$ & $\mathrm{R}_{4}=\mathrm{Et}$ & $4 \mathbf{i}$ & 2 & 97 & $94 / 6$ \\
\hline 10 & $\mathrm{R}_{1}=\mathrm{R}_{2}=\mathrm{H}, \mathrm{R}_{3}=\mathrm{CH}_{3}$ & $\mathrm{R}_{4}=\mathrm{Et}$ & $4 \mathbf{j}$ & 2 & 92 & $95 / 5$ \\
\hline 11 & $\mathrm{R}_{1}=\mathrm{R}_{2}=\mathrm{H}, \mathrm{R}=\mathrm{Br}$ & $\mathrm{R}_{4}=\mathrm{Et}$ & $4 \mathbf{k}$ & 5 & 70 & $92 / 8$ \\
\hline 12 & $\mathrm{R}_{1}=\mathrm{H}, \mathrm{R}_{2}=\mathrm{R}_{3}=\mathrm{F}$ & $\mathrm{R}_{4}=\mathrm{Et}$ & 41 & 3 & 88 & $99 / 1$ \\
\hline 13 & $\mathrm{R}_{1}=\mathrm{H}, \mathrm{R}_{2}=\mathrm{R}_{3}=\mathrm{Cl}$ & $\mathrm{R}_{4}=\mathrm{Et}$ & $4 \mathrm{~m}$ & 3 & 94 & $99 / 1$ \\
\hline 14 & $\mathrm{R}_{1}=\mathrm{R}_{2}=\mathrm{H}, \mathrm{R}_{3}=\mathrm{COOH}$ & $\mathrm{R}_{4}=\mathrm{Et}$ & $4 n$ & 3 & 91 & $99 / 1$ \\
\hline 15 & $\mathrm{R}_{1}=\mathrm{Et}, \mathrm{R}_{2}=\mathrm{R}_{3}=\mathrm{H}$ & $\mathrm{R}_{4}=\mathrm{Et}$ & 40 & 3 & 92 & $99 / 1$ \\
\hline 16 & $\mathrm{R}_{1}=$ benzyl, $\mathrm{R}_{2}=\mathrm{R}_{3}=\mathrm{H}$ & $\mathrm{R}_{4}=\mathrm{Et}$ & $4 p$ & 2 & 95 & $99 / 1$ \\
\hline 17 & $\mathrm{R}_{1}=\mathrm{R}_{2}=\mathrm{R}_{3}=\mathrm{H}$ & $\mathrm{R}_{4}=\mathrm{NH}_{2}$ & $\mathbf{4 q}$ & 2 & 64 & $98 / 2$ \\
\hline 18 & $\mathrm{R}_{1}=\mathrm{R}_{2}=\mathrm{H}, \mathrm{R}_{3}=\mathrm{CH}_{3}$ & $\mathrm{R}_{4}=\mathrm{NH}_{2}$ & $4 \mathbf{r}$ & 2 & 51 & $85 / 5$ \\
\hline
\end{tabular}

${ }^{\mathrm{a}}$ The reaction was carried out in $0.2 \mathrm{mmol}$ scale in 1,4-dioxane $(1 \mathrm{~mL})$ at $60{ }^{\circ} \mathrm{C}$, and the ratio of $\mathbf{1} / \mathbf{2} / \mathbf{3}$ is $1: 1: 1{ }^{\mathrm{b}}$ Isolated yield based on substituted isatins. ${ }^{\mathrm{c}}$ The $\mathrm{dr}$ refers to the diastereoselectivity and was determined by ${ }^{1} \mathrm{H}-\mathrm{NMR}$.

\section{Experimental}

\subsection{General}

All chemicals were obtained from commercial sources and used without further purification. Column chromatography was carried out on silica gel (300-400 mesh, Qingdao Marine Chemical Ltd., Qingdao, China). Thin layer chromatography (TLC) was performed on TLC silica gel 60 F254 plates. ${ }^{1} \mathrm{H}-\mathrm{NMR}$ and ${ }^{13} \mathrm{C}-\mathrm{NMR}$ spectra were recorded on Varian Gemini 400 Bruker AVII-400 or Bruker AVII-600 spectrometers. The chemical shifts were recorded in ppm relative to tetramethylsilane and with the solvent resonance as the internal standard. Data are reported as follows: chemical shift, multiplicity ( $\mathrm{s}=$ singlet, $\mathrm{d}=$ doublet, $\mathrm{t}=$ triplet, $\mathrm{m}=$ multiplet, $\mathrm{br}=$ broad), coupling constants $(\mathrm{Hz})$, integration. Chemical shifts are reported in ppm from the tetramethylsilane with the solvent resonance 
as internal standard. Mass Spectra (MS) were measured by 3200 Q TRAP LC/MS/MS utilizing electrospray ionization (ESI).

\subsection{Experimental Procedures}

A mixture of isatin $(0.2 \mathrm{mmol})$, L-proline (1 eq.), dimethyl maleate (1 eq.) in 1,4-dioxane ( $1 \mathrm{~mL})$ was stirred for $3 \mathrm{~h}$ at $60{ }^{\circ} \mathrm{C}$. After completion of the reaction (TLC), the solvent was removed under vacuum. The crude product was subjected to column chromatography on silica gel using $\mathrm{CH}_{2} \mathrm{Cl}_{2}$ - ethyl acetate $(2: 1)$ as the eluent to give $\mathbf{4 a}$ (60 $\mathrm{mg}$, 87\% yield). Compounds $\mathbf{4 b}-\mathbf{r}$ were synthesized by a similar procedure as described for compound 4a. For the separation of these compounds, the eluent of silica gel column chromatography consisted of appropriate mixtures of $\mathrm{CH}_{2} \mathrm{Cl}_{2}$ and ethyl acetate or $\mathrm{CH}_{2} \mathrm{Cl}_{2}$ and $\mathrm{MeOH}$.

\subsection{Spectral Data}

Dimethyl 2-oxo-1',2',5',6',7',7a'-hexahydrospiro[indoline-3,3'-pyrrolizine]-1',2'-dicarboxylate (4a). Yield 99\%; White solid; m.p. 194.5-197.8 ${ }^{\circ} \mathrm{C} ;{ }^{1} \mathrm{H}-\mathrm{NMR}\left(\mathrm{CDCl}_{3}, 400 \mathrm{MHz}\right): \delta 1.81-1.93$ (m, 2H), 1.95-2.01 (m, 2H), 2.49-2.52 (m, 1H), 3.10-3.16 (m, 1H), $3.39(\mathrm{~s}, 3 \mathrm{H}), 3.77(\mathrm{~s}, 3 \mathrm{H}), 3.88-3.96(\mathrm{~m}$, 2H), 4.26-4.32 (m, 1H), $6.88(\mathrm{~d}, J=7.2 \mathrm{~Hz}, 1 \mathrm{H}), 7.00-7.04(\mathrm{~m}, 1 \mathrm{H}), 7.23-7.25(\mathrm{~m}, 1 \mathrm{H}), 7.64(\mathrm{~d}$, $J=7.6 \mathrm{~Hz}, 1 \mathrm{H}), 8.26(\mathrm{~s}, 1 \mathrm{H}) ;{ }^{13} \mathrm{C}-\mathrm{NMR}\left(\mathrm{CDCl}_{3}, 100 \mathrm{MHz}\right): \delta 27.3,27.7,47.3,47.6,51.5,51.7,56.1$, $66.6,71.1,110.1,122.2,125.9,127.9,129.4,141.8,170.8,172.4,181.4$; HRMS: calcd. for $\mathrm{C}_{18} \mathrm{H}_{20} \mathrm{~N}_{2} \mathrm{O}_{5}^{+}[\mathrm{M}+\mathrm{H}]^{+}: 345.1472$, found: 345.1447 .

Dimethyl 5-methyl-2-oxo-1',2',5',6',7',7a'-hexahydrospiro[indoline-3,3'-pyrrolizine]-1',2'-dicarbo -xylate (4b). Yield 95\%; White solid; m.p. 187.2-189.6 ${ }^{\circ} \mathrm{C}$; ${ }^{1} \mathrm{H}-\mathrm{NMR}\left(\mathrm{CDCl}_{3}, 600 \mathrm{MHz}\right): \delta 1.88-1.92$ (m, $2 \mathrm{H}), 1.96-2.02(\mathrm{~m}, 2 \mathrm{H}), 2.31(\mathrm{~s}, 3 \mathrm{H}), 2.51-2.54(\mathrm{~m}, 1 \mathrm{H}), 3.16-3.20(\mathrm{~m}, 1 \mathrm{H}), 3.41(\mathrm{~s}, 3 \mathrm{H}), 3.77(\mathrm{~s}, 3 \mathrm{H})$, $3.81(\mathrm{~d}, J=7.8 \mathrm{~Hz}, 1 \mathrm{H}), 4.01-4.04(\mathrm{~m}, 1 \mathrm{H}), 4.29(\mathrm{q}, J=8.4 \mathrm{~Hz}, 1 \mathrm{H}), 6.80(\mathrm{~d}, J=7.8 \mathrm{~Hz}, 1 \mathrm{H}), 7.04(\mathrm{~d}$, $J=7.2 \mathrm{~Hz}, 1 \mathrm{H}), 7.37(\mathrm{~s}, 1 \mathrm{H}), 9.33(\mathrm{~s}, 1 \mathrm{H}) .{ }^{13} \mathrm{C}-\mathrm{NMR}\left(\mathrm{CDCl}_{3}, 150 \mathrm{MHz}\right): \delta 21.3,27.3,27.9,47.5$, 47.7, 51.5, 51.7, 55.4, 66.5, 71.2, 110.0, 12.6, 128.3, 129.8, 131.5, 139.5, 171.2, 172.2, 181.3; HRMS: calcd. for $\mathrm{C}_{19} \mathrm{H}_{22} \mathrm{~N}_{2} \mathrm{O}_{5}{ }^{+}[\mathrm{M}+\mathrm{H}]^{+}: 359.1529$, found: 359.1626 .

Dimethyl 5-bromo-2-oxo-1',2',5',6',7',7a'-hexahydrospiro[indoline-3,3'-pyrrolizine]-1',2'-dicarbo -xylate (4c). Yield 71\%; White solid; m.p. 119.3-121.6 ${ }^{\circ} \mathrm{C}$; ${ }^{1} \mathrm{H}-\mathrm{NMR}\left(\mathrm{CDCl}_{3}, 400 \mathrm{MHz}\right): \delta$ 1.71-1.78 $(\mathrm{m}, 1 \mathrm{H}), 1.85-1.92(\mathrm{~m}, 1 \mathrm{H}), 1.94-2.00(\mathrm{~m}, 2 \mathrm{H}), 2.47-2.50(\mathrm{~m}, 1 \mathrm{H}), 2.96-3.02(\mathrm{~m}, 1 \mathrm{H}), 3.43(\mathrm{~s}, 3 \mathrm{H})$, $3.78(\mathrm{~s}, 3 \mathrm{H}), 3.82-3.86(\mathrm{~m}, 1 \mathrm{H}), 3.93-3.95(\mathrm{~m}, 1 \mathrm{H}), 4.21-4.27(\mathrm{~m}, 1 \mathrm{H}), 6.79(\mathrm{~d}, J=8.0 \mathrm{~Hz}, 1 \mathrm{H}), 7.38$ $(\mathrm{d}, J=8.0 \mathrm{~Hz}, 1 \mathrm{H}), 7.83(\mathrm{~s}, 1 \mathrm{H}), 7.17(\mathrm{~s}, 1 \mathrm{H}) ;{ }^{13} \mathrm{C}-\mathrm{NMR}\left(\mathrm{CDCl}_{3}, 100 \mathrm{MHz}\right): \delta 27.4,27.5,46.7,47.3$, 51.7, 51.8, 56.6, 66.7, 70.7, 111.5, 115.1, 128.3, 131.0, 132.2, 140.7, 170.2, 172.1, 181.0; HRMS: calcd. for $\mathrm{C}_{18} \mathrm{H}_{19} \mathrm{BrN}_{2} \mathrm{O}_{5}{ }^{+}[\mathrm{M}+\mathrm{H}]^{+}:$423.0477, 425.0457, found: 423.0596, 425.0580.

Dimethyl 5,7-difluoro-2-oxo-1',2',5',6',7',7a'-hexahydrospiro[indoline-3,3'-pyrrolizine]-1',2'-dica -rboxylate (4d). Yield 90\%; White solid; m.p. 193.9-195.5 ${ }^{\circ} \mathrm{C}$; ${ }^{1} \mathrm{H}-\mathrm{NMR}\left(\mathrm{CDCl}_{3}, 600 \mathrm{MHz}\right): \delta$ 1.55-1.60 $(\mathrm{m}, 1 \mathrm{H}), 1.90-1.99(\mathrm{~m}, 3 \mathrm{H}), 2.44-2.47(\mathrm{~m}, 1 \mathrm{H}), 2.84-2.88(\mathrm{~m}, 1 \mathrm{H}), 3.42(\mathrm{~s}, 3 \mathrm{H}), 3.68-3.71(\mathrm{~m}, 1 \mathrm{H})$, $3.78(\mathrm{~s}, 3 \mathrm{H}), 4.09-4.10(\mathrm{~d}, J=8.4 \mathrm{~Hz}, 1 \mathrm{H}), 4.18-4.22(\mathrm{~m}, 1 \mathrm{H}), 6.81-6.84(\mathrm{~m}, 1 \mathrm{H}), 7.51(\mathrm{q}$, $J=8.4,1 \mathrm{H}), 8.21(\mathrm{~s}, 1 \mathrm{H}) ;{ }^{13} \mathrm{C}-\mathrm{NMR}\left(\mathrm{CDCl}_{3}, 150 \mathrm{MHz}\right): \delta 26.9,27.4,29.7,45.6,46.9,51.8,57.7$, 
66.7, 70.3, $104.6(\mathrm{dd}, J=28,21 \mathrm{~Hz}), 111.8(\mathrm{dd}, J=25,3 \mathrm{~Hz}), 124.9$ (dd, $J=12,3 \mathrm{~Hz}), 130.0$ (dd, $J=9,3 \mathrm{~Hz}), 146.1$ (dd, $J=244,11 \mathrm{~Hz}), 158.2$ (dd, $J=243,9 \mathrm{~Hz}), 169.6,172.3$, 179.9; HRMS: calcd. for $\mathrm{C}_{18} \mathrm{H}_{18} \mathrm{~F}_{2} \mathrm{~N}_{2} \mathrm{O}_{5}^{+}[\mathrm{M}+\mathrm{H}]^{+}: 381.1184$, found: 381.1279 .

Dimethyl 5,7-dichloro-2-oxo-1',2',5',6',7',7a'-hexahydrospiro[indoline-3,3'-pyrrolizine]-1',2'-dica -rboxylate (4e). Yield 89\%; White solid; m.p. 231.0-232.3 ${ }^{\circ} \mathrm{C}$; ${ }^{1} \mathrm{H}-\mathrm{NMR}\left(\mathrm{CDCl}_{3}, 400 \mathrm{MHz}\right): \delta$ 1.56-1.62 (m, 1H), 1.90-2.01 (m, 3H), 2.43-2.47 (m, 1H), $2.86(\mathrm{q}, J=7.6 \mathrm{~Hz}, 1 \mathrm{H}), 3.44(\mathrm{~s}, 3 \mathrm{H}), 3.69-3.73$ $(\mathrm{m}, 1 \mathrm{H}), 3.78(\mathrm{~s}, 3 \mathrm{H}), 4.04(\mathrm{~d}, J=8.0 \mathrm{~Hz}, 1 \mathrm{H}), 4.15-4.20(\mathrm{~m}, 1 \mathrm{H}), 7.27(\mathrm{~m}, 1 \mathrm{H}), 7.74(\mathrm{~d}, J=1.6 \mathrm{~Hz}$, $1 \mathrm{H}), 8.17(\mathrm{~s}, 1 \mathrm{H}) ;{ }^{13} \mathrm{C}-\mathrm{NMR}\left(\mathrm{CDCl}_{3}, 100 \mathrm{MHz}\right): \delta 26.9,27.3,45.6,46.9,51.8,57.6,66.6,70.8,115.2$, 126.8, 128.1, 128.9, 129.5, 137.8, 169.6, 172.1, 179.5; HRMS: calcd. for $\mathrm{C}_{18} \mathrm{H}_{18} \mathrm{Cl}_{2} \mathrm{~N}_{2} \mathrm{O}_{5}{ }^{+}[\mathrm{M}+\mathrm{H}]^{+}$: 413.0593, 415.0563, found: 413.0677, 415.0646.

1',2'-bis(methoxycarbonyl)-2-oxo-1',2',5',6',7',7a'-hexahydrospiro[indoline-3,3'-pyrrolizine]-5-carboxylic acid (4f). Yield 95\%; White solid; m.p. 250.0-253.0 ${ }^{\circ} \mathrm{C} ;{ }^{1} \mathrm{H}-\mathrm{NMR}$ (DMSO, $400 \mathrm{MHz}$ ): $\delta$ 1.84-1.92 $(\mathrm{m}, 4 \mathrm{H}), 2.31-2.34(\mathrm{~m}, 1 \mathrm{H}), 3.11-3.16(\mathrm{~m}, 1 \mathrm{H}), 3.40(\mathrm{~s}, 3 \mathrm{H}), 3.49-3.57(\mathrm{~m}, 1), 3.64(\mathrm{~d}$, $J=7.6 \mathrm{~Hz}, 1 \mathrm{H}), 3.70(\mathrm{~s}, 3 \mathrm{H}), 4.02-4.12(\mathrm{~m}, 1 \mathrm{H}), 6.83(\mathrm{~d}, J=8.0 \mathrm{~Hz}, 1 \mathrm{H}), 7.19(\mathrm{~d}, J=8.0 \mathrm{~Hz}, 1 \mathrm{H})$, $7.33(\mathrm{~s}, 1 \mathrm{H}), 10.43(\mathrm{~s}, 1 \mathrm{H}), 12.30(\mathrm{~s}, 1 \mathrm{H}) ;{ }^{13} \mathrm{C}-\mathrm{NMR}$ (DMSO, $\left.100 \mathrm{MHz}\right): \delta 27.1,27.8,41.1,47.6,51.7$, 51.8, 54.8, 65.9, 70.4, 109.8, 125.8, 128.3, 128.5, 130.8, 142.0, 171.3, 172.0, 173.4, 179.5; HRMS: calcd. for $\mathrm{C}_{19} \mathrm{H}_{2} \mathrm{~N}_{2} \mathrm{O}_{7}^{+}[\mathrm{M}+\mathrm{K}]^{+}: 427.1271$, found: 427.1264 .

Dimethyl 1-ethyl-2-oxo-1',2',5',6',7',7a'-hexahydrospiro[indoline-3,3'-pyrrolizine]-1',2'-dicarbox -ylate (4g). Yield 88\%; White solid; m.p. 98.6-100.1 ${ }^{\circ} \mathrm{C} ;{ }^{1} \mathrm{H}-\mathrm{NMR}\left(\mathrm{CDCl}_{3}, 400 \mathrm{MHz}\right): \delta 1.26$ (t, J=7.2 Hz, $3 \mathrm{H}), 1.80-1.88(\mathrm{~m}, 2 \mathrm{H}), 1.94-1.98(\mathrm{~m}, 2 \mathrm{H}), 2.42-2.45(\mathrm{~m}, 1 \mathrm{H}), 3.09-3.12(\mathrm{~m}, 1 \mathrm{H}), 3.35(\mathrm{~s}, 3 \mathrm{H})$, $3.67-3.72(\mathrm{~m}, 1 \mathrm{H}), 3.76(\mathrm{~s}, 3 \mathrm{H}), 3.78-3.94(\mathrm{~m}, 3 \mathrm{H}), 4.28(\mathrm{q}, J=8.0 \mathrm{~Hz}, 1 \mathrm{H}), 6.84(\mathrm{~d}, J=7.6 \mathrm{~Hz}, 1 \mathrm{H})$, $7.02(\mathrm{t}, J=3.6 \mathrm{~Hz}, 1 \mathrm{H}), 7.30(\mathrm{t}, J=8.0 \mathrm{~Hz}, 1 \mathrm{H}), 7.69(\mathrm{~d}, J=7.2 \mathrm{~Hz}, 1 \mathrm{H}) ;{ }^{13} \mathrm{C}-\mathrm{NMR}\left(\mathrm{CDCl}_{3}, 100 \mathrm{MHz}\right)$ : $\delta 12.4,27.3,27.5,34.8,47.1,47.5,51.4,51.6,56.4,66.6,70.3,108.1,122.1,125.7,127.8,129.3$, 143.5, 170.8, 172.4, 178.4; HRMS: calcd. for $\mathrm{C}_{20} \mathrm{H}_{24} \mathrm{~N}_{2} \mathrm{O}_{5}^{+}[\mathrm{M}+\mathrm{H}]^{+}: 373.1685$, found: 373.1772 .

Dimethyl 1-benzyl-2-oxo-1',2',5',6',7',7a'-hexahydrospiro[indoline-3,3'-pyrrolizine]-1',2'-dicarbo -xylate (4h). Yield 93\%; White solid; m.p. 138.4-139.8 ${ }^{\circ} \mathrm{C}$; ${ }^{1} \mathrm{H}-\mathrm{NMR}\left(\mathrm{CDCl}_{3}, 400 \mathrm{MHz}\right): \delta 1.73-1.78$ (m, 1H), 1.88-2.01 (m, 3H), 2.44-2.47 (m, 1H), 3.05-3.10 (m, 1H), $3.26(\mathrm{~s}, 3 \mathrm{H}), 3.78(\mathrm{~s}, 3 \mathrm{H}), 3.83(\mathrm{t}$, $J=8.0 \mathrm{~Hz}, 1 \mathrm{H}), 4.05(\mathrm{~d}, J=8.0 \mathrm{~Hz}, 1 \mathrm{H}), 4.30(\mathrm{q}, J=7.2 \mathrm{~Hz}, 1 \mathrm{H}), 4.76(\mathrm{~d}, J=15.6 \mathrm{~Hz}, 1 \mathrm{H}), 5.07(\mathrm{~d}$, $J=16.0 \mathrm{~Hz}, 1 \mathrm{H}), 6.67(\mathrm{~d}, J=7.6 \mathrm{~Hz}, 1 \mathrm{H}), 6.99(\mathrm{t}, J=7.6 \mathrm{~Hz}, 1 \mathrm{H}), 7.16(\mathrm{t}, J=7.6 \mathrm{~Hz}, 1 \mathrm{H}), 7.23-7.32$ $(\mathrm{m}, 5 \mathrm{H}), 7.77(\mathrm{~d}, J=7.6 \mathrm{~Hz}, 1 \mathrm{H}) ;{ }^{13} \mathrm{C}-\mathrm{NMR}\left(\mathrm{CDCl}_{3}, 100 \mathrm{MHz}\right): \delta 27.1,27.4,43.8,46.8,47.4,51.4$, 51.6, 57.3, 66.8, 70.2, 109.1, 122.4, 125.8, 127.1, 127.6, 127.7, 128.7, 129.2, 135.8, 143.4, 170.5, 172.5, 179.3; HRMS: calcd. for $\mathrm{C}_{25} \mathrm{H}_{26} \mathrm{~N}_{2} \mathrm{O}_{5}{ }^{+}[\mathrm{M}+\mathrm{H}]^{+}: 435.1842$, found: 435.1880 .

Diethyl 2-oxo-1',2',5',6',7',7a'-hexahydrospiro[indoline-3,3'-pyrrolizine]-1',2'-dicarboxylate (4i). Yield 97\%; White solid; m.p. 180.4-183.0 ${ }^{\circ} \mathrm{C}$; ${ }^{1} \mathrm{H}-\mathrm{NMR}\left(\mathrm{CDCl}_{3}, 400 \mathrm{MHz}\right): \delta 0.85$ (t, J=7.2 Hz, 3H), 1.32 $(\mathrm{t}, J=7.2 \mathrm{~Hz}, 3 \mathrm{H}), 1.81-1.91(\mathrm{~m}, 2 \mathrm{H}), 1.93-2.00(\mathrm{~m}, 2 \mathrm{H}), 2.46-2.50(\mathrm{~m}, 1 \mathrm{H}), 3.03-3.07(\mathrm{~m}, 1 \mathrm{H})$, $3.82-3.87(\mathrm{~m}, 3 \mathrm{H}), 3.93-3.96(\mathrm{~m}, 1 \mathrm{H}), 4.21-4.28(\mathrm{~m}, 3 \mathrm{H}), 6.89(\mathrm{~d}, J=7.6 \mathrm{~Hz}, 1 \mathrm{H}), 7.00(\mathrm{t}, J=7.6 \mathrm{~Hz}$, 1H), 7.21-7.27 (m, 1H), $7.72(\mathrm{~d}, J=7.6 \mathrm{~Hz}, 1 \mathrm{H}), 8.91(\mathrm{~s}, 1 \mathrm{H}) ;{ }^{13} \mathrm{C}-\mathrm{NMR}\left(\mathrm{CDCl}_{3}, 100 \mathrm{MHz}\right): \delta 13.6$, 
$14.3,27.3,27.5,47.1,47.5,56.5,60.6,66.7,71.1,110.0,122.2,126.2,128.2,129.3,141.8,170.1$, 171.9, 181.6; HRMS: calcd. for $\mathrm{C}_{20} \mathrm{H}_{24} \mathrm{~N}_{2} \mathrm{O}_{5}{ }^{+}[\mathrm{M}+\mathrm{H}]^{+}: 373.1685$, found: 373.1766 .

Diethyl 5-methyl-2-oxo-1',2',5',6',7',7a'-hexahydrospiro[indoline-3,3'-pyrrolizine]-1',2'-dicarbox -ylate (4j). Yield 92\%; White solid; m.p. 155.0-157.3 ${ }^{\circ} \mathrm{C} ;{ }^{1} \mathrm{H}-\mathrm{NMR}\left(\mathrm{CDCl}_{3}, 600 \mathrm{MHz}\right): \delta 0.88(\mathrm{t}, J=7.2 \mathrm{~Hz}$, $3 \mathrm{H}), 1.31(\mathrm{t}, J=7.2 \mathrm{~Hz}, 3 \mathrm{H}), 1.86-1.91(\mathrm{~m}, 2 \mathrm{H}), 1.97-2.02(\mathrm{~m}, 2 \mathrm{H}), 2.30(\mathrm{~s}, 3 \mathrm{H}), 2.50-2.52(\mathrm{~m}, 1 \mathrm{H})$, 3.11-3.15 (m, 1H), 3.83-3.95 (m, 4H), 4.22-4.28 (m, 3H), 6.79 (d, $J=7.8 \mathrm{~Hz}, 1 \mathrm{H}), 7.04(\mathrm{~d}, J=8.4 \mathrm{~Hz}$, 1H), 7.45 (s, 1H), 9.29 (s, 1H). ${ }^{13} \mathrm{C}-\mathrm{NMR}\left(\mathrm{CDCl}_{3}, 150 \mathrm{MHz}\right): \delta 13.6,14.3,21.3,27.3,27.7,47.4,47.6$, $55.9,60.51,60.52,66.6,71.2,109.9,125.9,128.6,129.6,131.4,139.5,170.5,171.8,181.6$; HRMS: calcd. for $\mathrm{C}_{21} \mathrm{H}_{26} \mathrm{~N}_{2} \mathrm{O}_{5}{ }^{+}[\mathrm{M}+\mathrm{H}]^{+}: 387.1842$, found: 387.1908 .

Diethyl 5-bromo-2-oxo-1',2',5',6',7',7a'-hexahydrospiro[indoline-3,3'-pyrrolizine]-1',2'-dicarbox -ylate (4k). Yield 70\%; White solid; m.p. 174.3-176.5 ${ }^{\circ} \mathrm{C} ;{ }^{1} \mathrm{H}-\mathrm{NMR}\left(\mathrm{CDCl}_{3}, 400 \mathrm{MHz}\right): \delta 0.89$ (t, $J=6.8 \mathrm{~Hz}$, $3 \mathrm{H}), 1.31(\mathrm{t}, J=7.2 \mathrm{~Hz}, 3 \mathrm{H}), 1.73-1.76(\mathrm{~m}, 1 \mathrm{H}), 1.83-1.90(\mathrm{~m}, 1 \mathrm{H}), 1.94-1.99(\mathrm{~m}, 2 \mathrm{H}), 2.45-2.48(\mathrm{~m}$, 1H), 2.92-2.98 (m, 1H), 3.74-3.78 (m, 1H), 3.83-3.88 (m, 1H), 3.92-3.96 (m, 2H), 4.20-4.27 (m, $3 \mathrm{H}), 6.78(\mathrm{~d}, J=8.4 \mathrm{~Hz}, 1 \mathrm{H}), 7.35-7.37(\mathrm{~m}, 1 \mathrm{H}), 7.89(\mathrm{~d}, J=1.6 \mathrm{~Hz}, 1 \mathrm{H}), 9.17(\mathrm{~s}, 1 \mathrm{H}) ;{ }^{13} \mathrm{C}-\mathrm{NMR}$ $\left(\mathrm{CDCl}_{3}, 100 \mathrm{MHz}\right): \delta 13.6,14.3,27.3,27.4,46.6,47.3,56.9,60.7,60.8,66.7,70.8,111.4,115.1$, 128.6, 131.2, 132.1, 140.8, 169.6, 171.6, 181.3; HRMS: calcd. for $\mathrm{C}_{20} \mathrm{H}_{23} \mathrm{BrN}_{2} \mathrm{O}_{5}{ }^{+}[\mathrm{M}+\mathrm{H}]^{+}: 451.0790$, 453.0770, found: $451.0746,453.0753$.

Diethyl 5,7-difluoro-2-oxo-1',2',5',6',7',7a'-hexahydrospiro[indoline-3,3'-pyrrolizine]-1',2'-dicarb -oxylate (4l). Yield 88\%; White solid; m.p. $119.3-121.8{ }^{\circ} \mathrm{C}$; ${ }^{1} \mathrm{H}-\mathrm{NMR}\left(\mathrm{CDCl}_{3}, 600 \mathrm{MHz}\right): \delta 0.90$ (t, $J=7.2 \mathrm{~Hz}, 3 \mathrm{H}), 1.33(\mathrm{t}, J=6.6 \mathrm{~Hz}, 3 \mathrm{H}), 1.54-1.56(\mathrm{~m}, 1 \mathrm{H}), 1.90-1.94(\mathrm{~m}, 2 \mathrm{H}), 1.95-2.00(\mathrm{~m}, 1 \mathrm{H})$, 2.44-2.46 (m, 1H), 2.80-2.84 (m, 1H), 3.60-3.63 (m, 1H), 3.84-3.92 (m, 2H), $4.10(\mathrm{~d}, J=8.4 \mathrm{~Hz}$, $1 \mathrm{H}), 4.17-4.20(\mathrm{~m}, 1 \mathrm{H}), 4.23-4.26(\mathrm{~m}, 2 \mathrm{H}), 6.80-6.84(\mathrm{~m}, 1 \mathrm{H}), 7.60(\mathrm{~d}, J=8.4 \mathrm{~Hz}, 1 \mathrm{H}), 7.66(\mathrm{~s}, 1 \mathrm{H})$; ${ }^{13} \mathrm{C}-\mathrm{NMR}\left(\mathrm{CDCl}_{3}, 150 \mathrm{MHz}\right): \delta$ 13.6, 14.4, 26.8, 27.4, 45.4, 46.9, 58.2, 60.7, 60.8, 66.8, 70.2, 104.4 $(\mathrm{dd}, J=28,22 \mathrm{~Hz}), 112.2(\mathrm{dd}, J=26,3 \mathrm{~Hz}), 124.9(\mathrm{dd}, J=12,3 \mathrm{~Hz}), 130.5$ (dd, $J=9,3 \mathrm{~Hz})$, $146.1(\mathrm{dd}, J=243,13 \mathrm{~Hz}), 158.3(\mathrm{dd}, J=242,10 \mathrm{~Hz}), 169.0,171.8,179.8$; HRMS: calcd. for $\mathrm{C}_{20} \mathrm{H}_{22} \mathrm{~F}_{2} \mathrm{~N}_{2} \mathrm{O}_{5}^{+}[\mathrm{M}+\mathrm{H}]^{+}:$409.1497, found: 409.1551 .

Diethyl 5,7-dichloro-2-oxo-1',2',5',6',7',7a'-hexahydrospiro[indoline-3,3'-pyrrolizine]-1',2'-dicarb -oxylate (4m). Yield 94\%; White solid; m.p. 197.6-199.1 ${ }^{\circ} \mathrm{C} ;{ }^{1} \mathrm{H}-\mathrm{NMR}\left(\mathrm{CDCl}_{3}, 600 \mathrm{MHz}\right): \delta 0.90$ (t, $J=7.2 \mathrm{~Hz}, 3 \mathrm{H}), 1.33(\mathrm{t}, J=7.2 \mathrm{~Hz}, 3 \mathrm{H}), 1.60-1.63(\mathrm{~m}, 1 \mathrm{H}), 1.90-2.00(\mathrm{~m}, 3 \mathrm{H}), 2.45-2.47(\mathrm{~m}, 1 \mathrm{H})$, $2.86(\mathrm{~m}, 1 \mathrm{H}), 3.66(\mathrm{t}, J=7.8 \mathrm{~Hz}, 1 \mathrm{H}), 3.84-3.87(\mathrm{~m}, 1 \mathrm{H}), 3.92-3.95(\mathrm{~m}, 1 \mathrm{H}), 4.07(\mathrm{t}, J=12.0 \mathrm{~Hz}$, $1 \mathrm{H}), 4.17-4.21(\mathrm{~m}, 1 \mathrm{H}), 4.23-4.27(\mathrm{~m}, 2 \mathrm{H}), 7.27-7.29(\mathrm{~m}, 1 \mathrm{H}), 7.82(\mathrm{~s}, 1 \mathrm{H}), 8.79(\mathrm{~s}, 1 \mathrm{H}) ;{ }^{13} \mathrm{C}-\mathrm{NMR}$ $\left(\mathrm{CDCl}_{3}, 150 \mathrm{MHz}\right): \delta 13.6,14.3,26.8,27.4,45.6,46.9,57.9,60.7,60.8,66.7,70.9,115.3,127.0$, 128.0, 128.7, 129.7, 138.1, 169.1, 171.6, 180.3; HRMS: calcd. for $\mathrm{C}_{20} \mathrm{H}_{22} \mathrm{Cl}_{2} \mathrm{~N}_{2} \mathrm{O}_{5}{ }^{+}[\mathrm{M}+\mathrm{H}]^{+}: 441.0906$, 443.0876, found: 441.0980, 443.0964 .

1',2'-bis(ethoxycarbonyl)-2-oxo-1',2',5',6',7',7a'-hexahydrospiro[indoline-3,3'-pyrrolizine]-5-carboxylic acid (4n). Yield 91\%; White solid; m.p. 228.1-230.6 ${ }^{\circ} \mathrm{C}$; ${ }^{1} \mathrm{H}-\mathrm{NMR}$ (DMSO, $600 \mathrm{MHz}$ ): $\delta 0.80$ (t, $J=7.2$ $\mathrm{Hz}, 3 \mathrm{H}), 1.21(\mathrm{t}, J=7.2 \mathrm{~Hz}, 3 \mathrm{H}), 1.71-1.76(\mathrm{~m}, 1 \mathrm{H}), 1.84-1.89(\mathrm{~m}, 2 \mathrm{H}), 2.23-2.26(\mathrm{~m}, 1 \mathrm{H}), 3.01(\mathrm{q}$, $J=7.2 \mathrm{~Hz}, 1 \mathrm{H}), 3.42-3.48(\mathrm{~m}, 1 \mathrm{H}), 3.63(\mathrm{~d}, J=8.4 \mathrm{~Hz}, 1 \mathrm{H}), 3.76-3.85(\mathrm{~m}, 3 \mathrm{H}), 4.00-4.02(\mathrm{~m}, 1 \mathrm{H})$, 
4.11-4.14 (m, 2H), $6.76(\mathrm{~d}, J=7.8 \mathrm{~Hz}, 1 \mathrm{H}), 7.13(\mathrm{~d}, J=7.8 \mathrm{~Hz}, 1 \mathrm{H}), 7.38(\mathrm{~s}, 1 \mathrm{H}), 10.38(\mathrm{~s}, 1 \mathrm{H}), 12.23$ (brs, $1 \mathrm{H}) ;{ }^{13} \mathrm{C}-\mathrm{NMR}$ (DMSO, $100 \mathrm{MHz}$ ): $\delta$ 13.9, 14.6, 27.2, 27.4, 47.2, 47.5, 55.6, 60.3, 60.4, 66.1, $70.4,109.7,126.2,128.1,128.8,130.6,142.1,170.4,171.6,173.2,179.9$; HRMS: calcd. for $\mathrm{C}_{21} \mathrm{H}_{24} \mathrm{~N}_{2} \mathrm{O}_{7}^{+}[\mathrm{M}+\mathrm{K}]^{+}: 455.1584$, found: 455.1568 .

Diethyl 1-ethyl-2-oxo-1',2',5',6',7',7a'-hexahydrospiro[indoline-3,3'-pyrrolizine]-1',2'-dicarboxyl -ate (4o). Yield 92\%; White solid; m.p. 66.1-68.4 ${ }^{\circ} \mathrm{C}$; ${ }^{1} \mathrm{H}-\mathrm{NMR}\left(\mathrm{CDCl}_{3}, 600 \mathrm{MHz}\right): \delta 0.81$ (t, $J=7.2 \mathrm{~Hz}$, $3 \mathrm{H}), 1.27(\mathrm{t}, J=7.2 \mathrm{~Hz}, 3 \mathrm{H}), 1.32(\mathrm{t}, J=7.2 \mathrm{~Hz}, 3 \mathrm{H}), 1.75-1.82(\mathrm{~m}, 1 \mathrm{H}), 1.85-1.90(\mathrm{~m}, 1 \mathrm{H})$, 1.95-1.97 (m, 2H), 2.40-2.43 (m, 1H), $3.05(\mathrm{q}, J=6.0 \mathrm{~Hz}, 1 \mathrm{H}), 3.69-3.72(\mathrm{~m}, 1 \mathrm{H}), 3.78-3.84(\mathrm{~m}$, $4 \mathrm{H}), 3.94(\mathrm{~d}, J=8.4 \mathrm{~Hz}, 1 \mathrm{H}), 4.22-4.27(\mathrm{~m}, 3 \mathrm{H}), 6.84(\mathrm{~d}, J=7.8 \mathrm{~Hz}, 1 \mathrm{H}), 7.00-7.03(\mathrm{~m}, 1 \mathrm{H})$, $7.28-7.30(\mathrm{~m}, 1 \mathrm{H}), 7.80(\mathrm{~d}, J=7.2 \mathrm{~Hz}, 1 \mathrm{H}) ;{ }^{13} \mathrm{C}-\mathrm{NMR}\left(\mathrm{CDCl}_{3}, 150 \mathrm{MHz}\right): \delta 12.5,13.5,14.3,27.2$, 27.3, 34.8, 46.8, 47.5, 56.9, 60.4, 60.5, 66.7, 70.2, 108.0, 122.0, 126.1, 128.0, 129.2, 143.5, 170.1, 172.0, 178.7; HRMS: calcd. for $\mathrm{C}_{22} \mathrm{H}_{28} \mathrm{~N}_{2} \mathrm{O}_{5}{ }^{+}[\mathrm{M}+\mathrm{H}]^{+}: 401.1998$, found: 401.2046 .

Diethyl 1-benzyl-2-oxo-1',2',5',6', 7',7a'-hexahydrospiro[indoline-3,3'-pyrrolizine]-1',2'-dicarboxy -late (4p). Yield 95\%; White solid; m.p. 97.2-99.3 ${ }^{\circ} \mathrm{C}$; ${ }^{1} \mathrm{H}-\mathrm{NMR}\left(\mathrm{CDCl}_{3}, 400 \mathrm{MHz}\right): \delta 0.66$ (t, $J=7.2 \mathrm{~Hz}$, $3 \mathrm{H}), 1.26-1.35(\mathrm{~m}, 3 \mathrm{H}), 1.71-1.78(\mathrm{~m}, 1 \mathrm{H}), 1.95-1.98(\mathrm{~m}, 3 \mathrm{H}), 2.41-2.46(\mathrm{~m}, 1 \mathrm{H}), 3.02(\mathrm{q}, J=8.0 \mathrm{~Hz}$, $1 \mathrm{H}), 3.71-3.78(\mathrm{~m}, 3 \mathrm{H}), 4.10(\mathrm{~d}, J=8.0 \mathrm{~Hz}, 1 \mathrm{H}), 4.24-4.29(\mathrm{~m}, 3 \mathrm{H}), 4.78(\mathrm{~d}, J=16.0 \mathrm{~Hz}, 1 \mathrm{H}), 5.05$ $(\mathrm{d}, J=15.6 \mathrm{~Hz}, 1 \mathrm{H}), 6.67(\mathrm{~d}, J=8.0 \mathrm{~Hz}, 1 \mathrm{H}), 6.97-7.00(\mathrm{~m}, 1 \mathrm{H}), 7.13-7.17(\mathrm{~m}, 1 \mathrm{H}), 7.26-7.31(\mathrm{~m}$, $5 \mathrm{H}), 7.85(\mathrm{~d}, J=7.2 \mathrm{~Hz}, 1 \mathrm{H}) ;{ }^{13} \mathrm{C}-\mathrm{NMR}\left(\mathrm{CDCl}_{3}, 100 \mathrm{MHz}\right): \delta 13.4,14.3,26.9,27.4,29.7,43.9,46.5$, 47.4, 57.6, 60.4, 60.5, 66.8, 70.1, 108.9, 122.4, 126.2, 127.2, 127.5, 127.9, 128.7, 129.0, 135.8, 143.5, 169.8, 172.0, 179.5; HRMS: calcd. for $\mathrm{C}_{27} \mathrm{H}_{30} \mathrm{~N}_{2} \mathrm{O}_{5}{ }^{+}[\mathrm{M}+\mathrm{H}]^{+}: 463.2155$, found: 463.2198 .

2-oxo-1',2',5',6',7',7a'-hexahydrospiro[indoline-3,3'-pyrrolizine]-1',2'-dicarboxamide (4q). Yield 64\%; White solid; m.p. 107.6-110.1 ${ }^{\circ} \mathrm{C}$; ${ }^{1} \mathrm{H}-\mathrm{NMR}$ (DMSO, $\left.600 \mathrm{MHz}\right): \delta 1.54-1.58(\mathrm{~m}, 1 \mathrm{H}), 1.79-1.83$ (m, 2H), 2.18-2.21 (m, 1H), 2.27-2.29 (m, 1H), 3.13-3.17 (m, 2H), 3.35-3.39 (m, 1H), 4.02-4.04 (m, 2H), $6.61(\mathrm{~s}, 1 \mathrm{H}), 6.79-6.91(\mathrm{~m}, 4 \mathrm{H}), 7.03(\mathrm{~s}, 1 \mathrm{H}), 7.51(\mathrm{~d}, J=8.4 \mathrm{~Hz}, 1 \mathrm{H}), 10.31(\mathrm{~s}, 1 \mathrm{H}) ;{ }^{13} \mathrm{C}-\mathrm{NMR}$ (DMSO, $150 \mathrm{MHz}$ ): $\delta 27.4,28.0,48.8,49.0,53.7,65.9,71.1,109.7,121.2,125.7,129.2,129.4,143.5$, 172.7, 172.8, 180.1; HRMS: calcd. for $\mathrm{C}_{16} \mathrm{H}_{18} \mathrm{~N}_{4} \mathrm{O}_{3}{ }^{+}[\mathrm{M}+\mathrm{H}]^{+}: 315.1379$, found: 315.1443 .

5-methyl-2-oxo-1',2',5',6',7',7a'-hexahydrospiro[indoline-3,3'-pyrrolizine]-1',2'-dicarboxamide (4r). Yield 51\%; White solid; m.p. 244.9-247.1 ${ }^{\circ} \mathrm{C} ;{ }^{1} \mathrm{H}-\mathrm{NMR}$ (DMSO, $400 \mathrm{MHz}$ ): $\delta$ 1.54-1.61 (m, 2H), $1.78-1.84(\mathrm{~m}, 3 \mathrm{H}), 2.22$ (s, 3H), 3.17 (d, $J=4.4 \mathrm{~Hz}, 2 \mathrm{H}), 3.99$ (d, $J=4.0 \mathrm{~Hz}, 2 \mathrm{H}), 6.60$ (s, 1H), 6.67 $(\mathrm{d}, J=7.6 \mathrm{~Hz}, 1 \mathrm{H}), 6.82(\mathrm{~d}, J=7.6 \mathrm{~Hz}, 2 \mathrm{H}), 7.01(\mathrm{~d}, J=8.0 \mathrm{~Hz}, 2 \mathrm{H}), 7.36(\mathrm{~s}, 1 \mathrm{H}), 10.15(\mathrm{~s}, 1 \mathrm{H})$; ${ }^{13} \mathrm{C}-\mathrm{NMR}(\mathrm{DMSO}, 100 \mathrm{MHz}): \delta$ 21.5, 27.4, 27.9, 48.7, 49.1, 54.1, 65.9, 71.1, 109.3, 125.8, 129.7 129.9, 141.0, 172.7, 172.8, 180.3; HRMS: calcd. for $\mathrm{C}_{17} \mathrm{H}_{20} \mathrm{~N}_{4} \mathrm{O}_{3}{ }^{+}[\mathrm{M}+\mathrm{H}]^{+}: 329.1535$, found: 329.1547 .

\section{Conclusions}

In this work, we have developed an efficient method for the synthesis of potentially biologically active spiropyrrolizidine oxindoles via a three-component 1,3-dipolar cycloaddition reaction. A range of spiropyrrolizidine oxindoles bearing two ester or two amide groups were obtained in high yields (up to $99 \%$ ) with excellent diastereoselectivities (up to 99:1 dr). The methodology is rapid, simple, and 
inexpensive affording complex compounds. Further study on the antibacterial, antiviral and antitumor activities of these compounds is underway. Supporting Information for this article is available online at http://www.mdpi.com/journal /molecules/. Included crystallographic data and molecular structure, ${ }^{1} \mathrm{H}-$, ${ }^{13} \mathrm{C}-\mathrm{NMR}$ and HRMS spectra of all compounds.

\section{Supplementary Materials}

Supplementary materials can be accessed at http://www.mdpi.com/1420-3049/16/10/8745/s1.

\section{Acknowledgements}

We appreciate the financial support of the Research Foundation for the Young Teachers of Sichuan University (2010SCU11033). We also thank Sichuan University Analytical \& Testing Center for the NMR analysis.

\section{References and Notes}

1. Usui, T.; Kondoh, M.; Cui, C.B.; Mayumi, T.; Osada, H. Tryprostatin A, a specific and novel inhibitor of microtubule assembly. Biochem. J. 1998, 333, 543-538.

2. Hilton, S.T.; Ho, T.C.; Pljevaljcic, G.; Jones, K. A new route to spirooxindoles. Org. Lett. 2000, 2, 2639-2641.

3. Marti, C.; Carreira, E.M. Construction of spiro[pyrrolidine-3,3-oxindoles]-recent applications to the synthesis of oxindole alkaloids. Eur. J. Org. Chem. 2003, 2003, 2209-2219.

4. Kumar, R.R.; Perumal, S.; Senthilkumar, P.; Yogeeswari, P.; Sriram, D. Discovery of antimycobacterial spiro-piperidin-4-ones: An atom economic, stereoselective synthesis, and biological intervention. J. Med. Chem. 2008, 51, 5731-5735.

5. Kumar, R.R.; Perumal, S.; Senthilkumar, P.; Yogeeswari, P.; Sriram, D. A facile synthesis and antimycobacterial evaluation of novel spiro-pyrido-pyrrolizines and pyrrolidines. Eur. J. Med. Chem. 2009, 44, 3821-3829.

6. Kumar, R.S.; Rajesh, S.M.; Perumal, S.; Banerjee, D.; Yogeeswari, P.; Sriram, D. Novel three-component domino reactions of ketones, isatin and amino acids: Synthesis and discovery of antimycobacterial activity of highly functionalised novel dispiropyrrolidines. Eur. J. Med. Chem. 2010, 45, 411-422.

7. Maheswari, S.U.; Balamurugan, K.; Perumal, S.; Yogeeswari, P.; Sriram, D. A facile 1,3-dipolar cycloaddition of azomethine ylides to 2-arylidene-1,3-indanediones: Synthesis of dispirooxindolylpyrrolothiazoles and their antimycobacterial evaluation. Bioorg. Med. Chem. Lett. 2010, 20, 7278-7282.

8. Prasanna, P.; Balamurugan, K.; Perumal, S.; Yogeeswari, P.; Sriram, D. A regio- and stereoselective 1,3-dipolar cycloaddition for the synthesis of novel spiropyrrolothiazolyloxindoles and their antitubercular evaluation. Eur. J. Med. Chem. 2010, 45, 5653-5661.

9. Karthikeyan, S.V.; Bala, B.D.; Raja, V.P.; Perumal, S.; Yogeeswari, P.; Sriram, D. A highly atom economic, chemo-, regio- and stereoselective synthesis and evaluation of spiro-pyrrolothiazoles as antitubercular agents. Bioorg. Med. Chem. Lett. 2010, 20, 350-353. 
10. Yong, S.R.; Ung, A.T.; Pyne, S.G.; Skelton, B.W.; White, A.H. Synthesis of novel 3'-spirocyclicoxindole derivatives and assessment of their cytostatic activities. Tetrahedron 2007, 63, 5579-5586.

11. Yu, S.; Qin, D.; Shangary, S.; Chen, J.; Wang, G.; Ding, K.; McEachern, D.; Qiu, S.; Nikolovska-Coleska, Z.; Miller, R.; Kang, S.; Yang, D.; Wang, S. Potent and orally active smallmolecule inhibitors of the MDM2-p53 interaction. J. Med. Chem. 2009, 52, 7970-7973.

12. Girgis, A.S. Regioselective synthesis of dispiro[1H-indene-2,3'-pyrrolidine-2',3"-[3H]indole]1,2"(1"H)-diones of potential anti-tumor properties. Eur. J. Med. Chem. 2009, 44, 91-100.

13. Silva, B.V.; Ribeiro, N.M.; Vargas, M.D.; Lanznaster, M.; Carneiro, J.W.; Krogh, R.; Andricopulo, A.D.; Dias, L.C.; Pinto, A.C. Synthesis, electrochemical studies and anticancer activity of ferrocenyl oxindoles. Dalton Trans. 2010, 39, 7338-7344.

14. Kamal, A.; Ramakrishna, G.; Raju, P.; Rao, A.V.; Viswanath, A.; Nayak, V.L.; Ramakrishna, S. Synthesis and anticancer activity of oxindole derived imidazo[1,5-a]pyrazines. Eur. J.Med. Chem. 2011, 46, 2427-2435.

15. Periyasami, G.; Raghunathan, R.; Surendiran, G.; Mathivanan, N. Synthesis of novel spiropyrrolizidines as potent antimicrobial agents for human and plant pathogens. Bioorg. Med. Chem. Lett. 2008, 18, 2342-23425.

16. Mhaske, P.C.; Shelke, S.H.; Jadhav, R.P.; Raundal, H.N.; Patil, S.V.; Patil, A.A.; Bobade, V.D. Synthesis, characterization, and antimicrobial activity of 3'-(4-(2-substituted thiazol-4yl)phenyl)spiro[indoline-3,2'-thiazolidine]-2,4'-diones. J. Heterocyclic Chem. 2010, 47, 1415-1420.

17. Prasanna, P.; Balamurugan, K.; Perumal, S.; Yogeeswari, P.; Sriram, D. A regio- and stereoselective 1,3-dipolar cycloaddition for the synthesis of novel spiropyrrolothiazolyloxindoles and their antitubercular evaluation. Eur. J. Med. Chem. 2010, 45, 5653-5661.

18. Raj, A.A.; Raghunathan, R.; Sridevi Kumari, M.R.; Raman, N. Synthesis, antimicrobial and antifungal activity of a new class of spiro pyrrolidines. Bioorg. Med. Chem. 2003, 11, 407-419.

19. Thangamani, A. Regiospecific synthesis and biological evaluation of spirooxindolopyrrolizidines via [3+2] cycloaddition of azomethine ylide. Eur. J. Med. Chem. 2010, 45, 6120-6126.

20. Jiang, T.; Kuhen, K.L.; Wolff, K.; Yin, H.; Bieza, K.; Caldwell, J.; Bursulaya, B.; Wu, T.Y.; He, Y. Design, synthesis and biological evaluations of novel oxindoles as HIV-1 non-nucleoside reverse transcriptase inhibitors. Part I. Bioorg. Med. Chem. Lett. 2006, 16, 2105-2108.

21. Jiang, T.; Kuhen, K.L.; Wolff, K.; Yin, H.; Bieza, K.; Caldwell, J.; Bursulaya, B.; Tuntland, T.; Zhang, K.; Karanewsky, D.; He, Y. Design, synthesis, and biological evaluations of novel oxindoles as HIV-1 non-nucleoside reverse transcriptase inhibitors. Part 2. Bioorg. Med. Chem. Lett. 2006, 16, 2109-2112.

22. Kornet, M.J.; Thio, A.P. Oxindole-3-spiropyrrolidines and piperidines. Synthesis and local anesthetic activity. J. Med. Chem. 1976, 19, 892-898.

23. Ardill, H.; Dorrity, M.J.R.; Grigg, R.; Leon-Ling, M.-S.; Malone, J.F.; Sridharan, V.; Thianpatanagul, S. X=Y-ZH compounds as potential 1,3-dipoles. Part $28^{1,2}$ the iminium ion route to azomethine ylides. background and reaction of amines with bifunctional ketones. Tetrahedron 1990, 46, 6433-6448. 
24. Ardill, H.; Grigg, R.; Sridharan, V.; Surendrakumar, S. X=Y-ZH Systems as potential 1,3-dipoles: Part 19. Intramolecular cycloadditions of non-stabilised azomethine ylides generated via the decarboxylative route from $\alpha$ - amino acids. Tetrahedron 1988, 44, 4953-4966.

25. Ganguly, A.K.; Seah, N.; Popov, V.; Wang, C.H.; Kuang, R.; Saksena, A.K.; Pramanik, B.N.; Chan, T.M.; McPhail, A.T. Solution- and solid-phase synthesis of enantiomerically pure spiro oxindoles. Tetrahedron Lett. 2002, 43, 8981-8983.

26. Lo, M.M.C.; Neumann, C.S.; Nagayama, S.; Perlstein, E.O.; Schreiber, S.L. A library of spirooxindoles based on a stereoselective three-component coupling reaction. J. Am. Chem. Soc. 2004, 126, 16077-16086.

27. Teng, D.; Zhang, H.; Mendonca, A. An efficient synthesis of a spirocyclic oxindole analogue. Molecules 2006, 11, 700-706.

28. Ding, K.; Lu, Y.; Nikolovska-Coleska, Z.; Wang, G.; Qiu, S.; Shangary, S.; Gao, W.; Qin, D.; Stuckey, J.; Krajewski, K.; Roller, P.P.; Wang, S. Structure-based design of spiro-oxindoles as potent, specific small-molecule inhibitors of the MDM2-p53 interaction. J. Med. Chem. 2006, 49, 3432-3435.

29. Miyamoto, H.; Okawa, Y.; Nakazaki, A.; Kobayashi, S. Highly diastereoselective one-pot synthesis of spirocyclic oxindoles through intramolecular Ullmann coupling and Claisen rearrangement. Angew. Chem. Int. Ed. 2006, 45, 2274-2277.

30. Basavaiah, D.; Reddy, K.R. Simple and one-pot protocol for synthesis of indene-spiro-oxindoles involving tandem Prins and Friedel-Crafts reactions. Org. Lett. 2007, 9, 57-60.

31. Bencivenni, G.; Wu, L.Y.; Mazzanti, A.; Giannichi, B.; Pesciaioli, F.; Song, M.P.; Bartoli, G.; Melchiorre, P. Targeting structural and stereochemical complexity by organocascade catalysis: Construction of spirocyclic oxindoles having multiple stereocenters. Angew. Chem. Int. Ed. 2009, 48, 7200-7203.

32. Shanthi, G.; Perumal, P.T. An efficient one-pot synthesis of novel pyrazolophthalazinyl spirooxindoles. J. Chem. Sci. 2010, 122, 415-421.

33. Hazra, A.; Paira, P.; Sahu, K.B.; Naskar, S.; Saha, P.; Paira, R.; Mondal, S.; Maity, A.; Luger, P.; Weber, M.; Mondal, N.B.; Banerjee, S. Chemistry of andrographolide: formation of novel di-spiropyrrolidino and di-spiropyrrolizidino-oxindole adducts via one-pot three-component [3+2] azomethine ylide cycloaddition. Tetrahedron Lett. 2010, 51, 1585-1588.

34. Gomes, P.J.S.; Nunes, C.M.; Pais, A.A.C.C.; Pinho e Melo, T.M.V.D.; Arnaut, L.G. 1,3-Dipolar cycloaddition of azomethine ylides generated from aziridines in supercritical carbon dioxide. Tetrahedron Lett. 2006, 47, 5475-5479.

35. Grigg, R.; Sarker, M.A.B. XY-ZH compounds as potential 1,3-dipoles. Part 63: Silver catalysed azomethine ylide cycloaddition-the synthesis of spiro homoserine lactone analogues. Tetrahedron 2006, 62, 10332-10343.

36. Watson, A.A.; Fleet, G.W.J.; Asano, N.; Molyneux, R.J.; Nash, R.J. Polyhydroxylated alkaloidsnatural occurrence and therapeutic applications. Phytochemistry 2001, 56, 265-295.

37. Shanmugam, P.; Viswambharan, B.; Madhavan, S. Synthesis of novel functionalized 3spiropyrrolizidine and 3-spiropyrrolidine oxindoles from Baylis-Hillman adducts of isatin and heteroaldehydes with azomethine ylides via [3+2] cycloaddition. Org. Lett. 2007, 9, 4095-4098. 
38. Yu, J.; He, L.; Chen, X.H.; Song, J.; Chen, W.J.; Gong, L.Z. Highly enantioselective catalytic 1,3-dipolar cycloaddition involving 2,3-allenoate dipolarophiles. Org. Lett. 2009, 11, 4946-4949.

39. Cheng. M.N.; Wang, H.; Gong, L.Z. Asymmetic organocatalytic 1,3-dipolar cycloaddition of azomethine ylide to methyl 2-(2-nitrophenyl)acrylate for the synthesis of diastereoisomers of spirotryprostatin A. Org. Lett. 2011, 13, 2418-2421.

40. Michael, J.P. Indolizidine and quinolizidine alkaloids. Nat. Prod. Rep. 1997, 14, 619-636.

41. Liddell, J.R. Pyrrolizidine alkaloids. Nat. Prod. Rep. 1998, 15, 363-370.

42. Rehn, S.; Bergman, J.; Stensland, B. The three-component reaction between isatin, $\alpha$-amino acids, and dipolarophiles. Eur. J. Org. Chem. 2004, 2004, 413-418.

43. Prasanna, R.; Purushothaman, S.; Raghunathan, R. Highly regioselective synthesis of glycospiro heterocycles through 1,3-dipolar cycloaddition reaction. Tetrahedron Lett. 2010, 51, 4538-4542.

44. Crystallographic data of $\mathbf{4 a}$ reported in this manuscript have been deposited with Cambridge Crystallographic Data Centre as supplementary publication no. CCDC-828257. Copies of the data can be obtained free of charge via www.ccdc.cam.ac.uk/conts/retrieving.html (or from the Cambridge Crystallographic Data Centre, 12, Union Road, Cambridge, CB2 1EZ, UK; fax: +44 1223 336033; or deposit@ccdc.cam.ac.uk).

Sample Availability: Samples are available from the authors.

(C) 2011 by the authors; licensee MDPI, Basel, Switzerland. This article is an open access article distributed under the terms and conditions of the Creative Commons Attribution license (http://creativecommons.org/licenses/by/3.0/). 